\title{
Die allgemeine Wehrpflicht in Deutschland - zur aktuellen Debatte um die Wehrform in unserem Land
}

\author{
Ulrich Kirsch*
}

\section{Die aktuelle Debatte um die Wehrpflicht}

In einer Grundsatzrede am 26. Mai 2010 an der Führungsakademie der Bundeswehr in Hamburg hat der Bundesminister der Verteidigung, Karl-Theodor zu Guttenberg, die Frage der Wehrpflicht als „Gretchenfrage“ tituliert. ${ }^{1}$ Seit Bundeskanzlerin Angela Merkel und Vizekanzler Guido Westerwelle die Ergebnisse der Kabinettsklausur von Meseberg am 7. Juni 2010 vorgestellt haben, ist diese „Gretchenfrage“ zur Wehrpflicht gestellt. Es bleibt abzuwarten, ob die Debatte tatsächlich anhand sicherheits- und verteidigungspolitischer Erwägungen geführt wird oder aber doch die Haushaltlage das Ergebnis bestimmt. Tatsache ist, die Wehrpflicht als Wehrform in Deutschland steht wieder einmal zur Diskussion.

Seit Ende des Kalten Krieges ist die Debatte um die allgemeine Wehrpflicht in Deutschland immer wieder entbrannt. Dabei konnten, teils befördert durch parteipolitische Positionen, teils durch sicherheitspolitische Diskussionen um die Ausrichtung der Bundeswehr, Wellenbewegungen beobachtet werden, in denen das Thema mehr oder weniger im Fokus des öffentlichen Interesses stand. Vor allem die seit den 1990er Jahren zunehmende Aussetzung oder Abschaffung der allgemeinen Wehrpflicht in den Mitgliedstaaten der NATO und der EU haben auch in Deutschland immer wieder zur Diskussion um die zukünftige Ausgestaltung unserer Wehrform geführt. ${ }^{2}$ Dabei lässt sich jedoch beobachten, dass die Erfahrungen mit diesem Schritt keineswegs nur positiv waren.

In jüngsten Veröffentlichungen wird nach Untersuchung der Erfahrungen, die diese Länder mit der Abschaffung der Wehrpflicht gemacht haben, davon abgeraten, diesen Schritt auch für Deutschland zu vollziehen, oder aber auf schwerwiegende negative Folgen hingewiesen, welche die Streitkräfte der Länder zu verzeichnen hätten, die sich dazu entschieden haben. ${ }^{3}$ Weiterhin ist die Wehrpflicht bei der deutschen Bevölkerung nach wie vor hoch angesehen. So ist die Einstellung zur Wehrpflicht bei der deutschen Bevölkerung mit 70 Prozent der Befragten eher positiv bis sehr positiv. Eine überwiegende Mehrheit - 62 Prozent der Bundesbürger -, sprach sich bei einer Befragung im Jahr 2008 für den Erhalt der allgemeinen Wehrpflicht aus.

* Ulrich Kirsch ist Bundesvorsitzender des Deutschen BundeswehrVerbandes (DBwV).

1 Vgl. dazu: Grundsatzrede des Bundesministers der Verteidigung, Dr. Karl-Theodor Freiherr zu Guttenberg, anlässlich des Besuches der Führungsakademie der Bundeswehr und der Kommandeurtagung der Streitkräftebasis am 26. Mai 2010 in Hamburg.

2 Aktuell verfügen 17 von 28 NATO-Mitgliedstaaten und 15 von 27 EU-Mitgliedern über Freiwilligenarmeen. Auf eine Einberufung von Wehrpflichtigen verzichten aktuell 22 von 28 NATO-Staaten und 19 von 27 EU Mitgliedsländern. Vgl. dazu Buch, D. (2010). Wohin mit der Wehrpflicht? Weisen die Partner wirklich den richtigen Weg? Tabelle: Übersicht über die Wehrstrukturentscheidungen in Nato und EU, S. 84/86.

3 Vgl. dazu Buch, D. (2010). aaO., S. 82. Vgl. dazu auch: Der Tagesspiegel (2007). Wehrpflicht im europäischen Ausland. http://www.tagesspiegel.de/politik/ international/Wehrpflicht;art123,2370188 (06.02.2010).
Hier konnte sogar ein Anstieg bei der Zustimmung seit 2005 verzeichnet werden, als sich nur 53 Prozent der Bundesbürger für die Beibehaltung der Wehrpflicht ausgesprochen hatten. ${ }^{4}$

Die Wehrpflicht jetzt anhand der Kassenlage im Bundeshaushalt zu beurteilen, hält der Deutsche BundeswehrVerband (DBwV) für äußerst fragwürdig. Die Verantwortung der Politik für die Sicherheit Deutschlands und seiner Bürger, aber auch die Verantwortung als verlässlicher Bündnispartner machen es zwingend notwendig, eine an den Interessen Deutschlands orientierte, grundlegende Debatte über die Wehrpflicht zu führen.

Dabei können durchaus verschiedene Konzepte der Wehrpflicht in die Diskussion eingebracht werden. Dies kann auch eine Auseinandersetzung mit dem von der so genannten „Weizsäcker-Kommission“ im Jahr 2000 eingebrachten Vorschlag beinhalten, eine so genannte Auswahlwehrpflicht einzuführen. ${ }^{5}$

Zunächst sollen allerdings Gründe aufgezeigt werden, warum der DBwV die Wehrpflicht auch aktuell und vor dem Hintergrund der haushalterischen Debatte für sicherheitspolitisch notwendig, militärisch sinnvoll und gesellschaftlich wünschenswert hält. Zum Ende des Beitrags wird dann, in der gebotenen Kürze, auf den Vorschlag und die mögliche Ausgestaltung einer Auswahlwehrpflicht, aus Sicht des DBwV, eingegangen.

\section{Warum wir die allgemeine Wehrpflicht brauchen}

\section{a. Die allgemeine Wehrpflicht ist sicherheits- politisch unverzichtbar}

Mit dem Ende des Kalten Krieges ist keineswegs, wie zwar auch in Deutschland erhofft, dass „Ende der Geschichte“ zu verzeichnen gewesen. Totalitäre Systeme wurden nicht von sogenannten „liberalen Demokratien“ abgelöst; und wir sind im 21. Jahrhundert vielleicht weiter von einer globalen Friedensordnung entfernt, als wir dies im 20. Jahrhundert noch waren. ${ }^{6}$ Vielmehr stehen wir vor neuen Bedrohungen und Risiken. Der internationale Terrorismus, die Proliferation und illegaler Waffenhandel, regionale Konflikte und fragile Staatlichkeit sind Bestandteile dieser neuen Bedrohungen. Sie werden

4 Vgl. dazu Bulmann, Th. (2008). Bevölkerungsbefragung 2008. Sicherheitsund verteidigungspolitisches Meinungsklima in Deutschland. Kurzbericht, S. 37/39.

5 Vgl. dazu: Kommission „Gemeinsame Sicherheit und Zukunft der Bundeswehr" (2000). Bericht der Kommission an die Bundesregierung. Bundesminsterium der Verteidigung. Berlin

6 Vgl. dazu Fukuyama, F. (1992). Das Ende der Geschichte. Wo stehen wir? München. 
ergänzt durch Pandemien und Seuchen, die im Rahmen einer fortschreitenden Globalisierung keiner Begrenzung durch Staaten mehr unterliegen. Als stark exportorientierte und dabei rohstoffarme Mittelmacht in Europa sind wir zudem stark von einer dauerhaften Rohstoff- und Energiesicherheit sowie von sicheren Transportwegen abhängig. Als wirtschaftlich hoch entwickelte Nation üben wir zudem eine hohe Anziehungskraft für Migranten aus aller Welt aus. ${ }^{7}$

Diese aktuellen Bedrohungen und Risiken für Deutschland betreffen alle Bürgerinnen und Bürger. Somit kann die Abwehr auch nur als Gemeinschaftaufgabe gelingen. Dazu kommt, dass Deutschland in der westlichen Wertegemeinschaft mit seiner demokratischen Lebensform eine zentrale Rolle einnimmt. Das Einstehen für diese Art der Gemeinschaft ist deshalb auch Aufgabe aller Bürger. Die allgemeine Wehrpflicht kann hier einen wesentlichen Beitrag zur Sicherheitsvorsorge unseres Landes leisten.

Die Bundesrepublik Deutschland hat mehr als 40 Jahre, als der Staat an der Nahtstelle des Ost-West Konflikts, vom transatlantischen Bündnis profitiert. Auch heute bietet die Einbettung in internationale Sicherheitsstrukturen einen wesentlichen Aspekt des Schutzes unseres Staates. Bündnisse beruhen jedoch ganz wesentlich darauf, dass sich ihre Mitglieder ihren Fähigkeiten entsprechend einbringen. Aufgrund seiner Größe, Bevölkerungszahl und wirtschaftlichen Leistungsfähigkeit steht Deutschland immer wieder im Fokus der internationalen Betrachtung, wenn es um das Einbringen dieser Fähigkeiten im Rahmen internationaler Bündnisse geht. Deutschland hat seit Beginn der 1990er Jahre die Bundeswehr auf 252.500 Soldaten reduziert.

Legt man den durchschnittlichen Umfang der Streitkräfte aller NATO Staaten im Verhältnis zu ihrer Bevölkerung zu Grunde (0,5 Prozent), so müsste der Umfang der Bundeswehr in Deutschland etwa 400.000 Soldaten betragen. ${ }^{8}$ Selbst wenn man davon ausgeht, dass diese Zahl innenpolitisch nicht durchsetzbar erscheint, so lässt sich doch feststellen, dass ein deutscher Streitkräfteumfang unter 250.000 Soldatinnen und Soldaten unseren Bündnispartnern schwer vermittelbar wäre. ${ }^{9}$ Zumal sich Deutschland auch bei der Finanzierung seiner Streitkräfte seit Jahren im unteren Bereich der NATO-Partner bewegt und im Jahr 2008 mit 1,3 Prozent des BIP im Bereich von Ungarn und Litauen anzusiedeln war. Vom im Rahmen der NATO formulierten Ziel, zwei Prozent des BIP für die Verteidigung aufzuwenden, ist man damit in Deutschland weiter entfernt denn je. ${ }^{10}$ Dieses Verhältnis würde sich mit einer nun

7 Vgl. dazu Bundesministerium der Verteidigung (2006). Weißbuch 2006. Zur Sicherheitspolitk Deutschlands und zur Zukunft der Bundeswehr. Berlin, S. $19 / 23$.

8 Vgl. dazu Mey, H. (2003). Gibt es heute eine vermittelbare sicherheitspolitische Legitimation für die Allgemeine Wehrpflicht? In: Prüfert, A. (Hrsg.), Hat die allgemeine Wehrpflicht in Deutschland eine Zukunft? Baden-Baden, S. 48. Vgl. dazu auch Wittmann, K. (2010). Für den Erhalt der allgemeinen Wehrpflicht. Die Bundeswehr, 2010 (2), S. 8.

9 Vgl. dazu Schnell, J. (2010). Neue Entscheidung zur allgemeinen Wehrpflicht - Zur Verkürzung des Grundwehrdienstes von 9 Monoate auf 6 Monate sowie zur Umwandlung der Bundeswehr in eine Freiwilligenarmee. Diskussionsbeitrag, Universität der Bundeswehr München, S. 18.

10 Basierend auf festen Preisen gab der Durchnitt aller NATO-Mitglieder im Jahr 2008 2,8 Prozent des BIP für Verteidigung aus. Deutschland hat im Jahr 2008 1,3 Prozent seines BIP für die Verteidigung aufgewendet. Vgl. dazu NATO (2009). Financial and Economic Data Relating to NATO Defence. Press Release 19.02.2009, S. 6. diskutierten Abschaffung oder Aussetzung der Wehrpflicht und einer Reduzierung der Bundeswehr auf 150.000 Zeit- und Berufssoldaten noch dramatisch verschlechtern. ${ }^{11}$

Vor dem Hintergrund der demografischen Entwicklung und der sicherheitspolitischen Handlungsfähigkeit gewährleistet die allgemeine Wehrpflicht einen Umfang deutscher Streitkräfte, der seinen Bündnisverpflichtungen gerecht wird.

Das Oderhochwasser 1997, das Elbehochwasser 2002 und die Schneekatastrophe in Bayern im Jahr 2006, um nur einige Beispiele zu nennen, haben gezeigt, dass zur Bewältigung nationaler Katastrophen der Einsatz der Bundeswehr im Rahmen der Amtshilfe unverzichtbar ist. Dabei standen wir in Deutschland noch nicht vor der Bewährungsprobe terroristischer Anschläge, wie sie in Madrid 2004 oder aber in London im Jahr 2005 zu beklagen waren. Auch wenn im Rahmen besonders schwerer Unglücksfälle, wie dem Zugunglück von Eschede im Jahr 1998, die Bundeswehr bereits ihre Leistungsfähigkeit unter Beweis gestellt hat. Um die besonderen nationalen Aufgaben, die im Zuge von Terroranschlägen, Naturkatastrophen und besonders schweren Unglücksfällen auftreten können, bewältigbar zu machen, ist die Rückgriffmöglichkeit auf die Bundeswehr und insbesondere auch ihre Wehrdienstleistenden unverzichtbar.

Das 21. Jahrhundert ist nicht nur, wie bereits zu Beginn erwähnt, von einem sicherheitspolitischen Paradigmenwechsel geprägt, auch die Geschwindigkeit der Veränderungen nimmt stetig zu. Die wichtigste Aufgabe des Staates bleibt dabei jedoch konstant, nämlich die Sicherheit seiner Bürgerinnen und Bürger zu gewährleisten und dafür die notwendigen Vorkehrungen zu treffen. Wie schnell sich das Sicherheitsumfeld ändern kann, hat gerade der Beginn des 21. Jahrhunderts gezeigt. Wer hätte im Zuge der Jahrhundertwende die Auseinandersetzung im Kosovo 1999, wo ethnische Vertreibung wieder zum Mittel der Politik zu werden drohte, die Terroranschläge des 11. September 2001 in den USA, in deren Zusammenhang erstmalig der Bündnisfall der NATO nach Art. 5 der Charta ausgerufen wurde, oder die Auseinandersetzungen im August 2008 zwischen Georgien und Russland, in denen sich ein Beitrittskandidat der NATO in einer militärischen Konfliktsituation mit Russland wiederfand, in dieser Form vorhergesehen? Um diesen schnellen und unvorhersehbarer werdenden Veränderungen auch flexibel und zeitgerecht begegnen zu können, bietet die allgemeine Wehrpflicht ein wesentliches Instrumentarium. Gerade die Rekonstitutionsfähigkeit versetzt Deutschland in die Lage, sicherheitspolitischen Risiken reaktiv, präventiv und risikoorientiert begegnen zu können. Im Krisen- und Konfliktfall ist der zeitgerechte Aufbau der Verteidigungsfähigkeit Deutschlands wesentlich, um den Schutz Deutschlands und seiner Bürger zu gewährleisten. Die allgemeine Wehrpflicht stellt dabei die strategische Komponente und das effizienteste Mittel dar, dieses Aufwuchspotenzial bereitzustellen. Weiterhin ist die allgemeine Wehrpflicht in der Lage, über ein zeitgemäßes Reservistensystem zur Sicherheitsvorsorge unseres Landes wesentlich mit

11 Ein Umfang der Bundeswehr von 150.000 Soldatinnen und Soldaten entspricht einem Bevölkerungsanteil von 0,18 Prozent. Der diskutierte mittelfristige Verteidigunshaushalt in Höhe von 25 Milliarden Euro bedeutet einen Anteil am BIP Deutschlands von 1,0 Prozent, der im NATO-Vergleich in etwa dem Beitrag Belgiens und in Europa dem des Nicht-NATO-Mitglieds Österreich entspäche. 
beizutragen. Eine Aussetzung der Wehrpflicht würde de facto das Ende der allgemeinen Wehrpflicht bedeuten, denn eine Reaktivierung erscheint, auch vor dem Hintergrund des damit verbundenen Eskalationspotenzials in einer Krise, schwierig umsetzbar. ${ }^{12}$ Im internationalen Vergleich lässt sich überdies kein Beispiel in der jüngeren Vergangenheit finden, bei dem ein Land, das die Wehrpflicht ausgesetzt oder abgeschafft hat, diese wieder einführen konnte. ${ }^{13}$

\section{b. Die allgemeine Wehrpflicht ist militärisch sinnvoll}

Zeitgemäße Streitkräfte definieren sich zunehmend durch die Verknüpfung ziviler und militärischer Fähigkeiten und Kenntnisse. Die Vielfalt der benötigten Fähigkeiten kann nicht allein innerhalb der Streitkräfte erworben werden. Die allgemeine Wehrpflicht garantiert dabei einen permanenten Personalaustausch, um aktuelle gesellschaftliche und berufsspezifische Entwicklungen auch in der Bundeswehr abzubilden. ${ }^{14}$ Sinnvollerweise würde natürlich die zukünftige Ausgestaltung der Wehrpflicht diesem Aspekt noch stärker Rechnung tragen, indem sie beispielsweise mit einem Reservistenkonzept aufwartet, das auch Wehrpflichtige bewusst mit einbindet. Es erscheint auch notwendig, um das Potenzial der zivilen Fähigkeiten bewusst ausschöpfen zu können, dass alle gesellschaftlichen Schichten unter den Wehrpflichtigen vertreten sind. Eine gelungene Ausgestaltung der Wehrpflicht kann dies gewährleisten. Zumal die Jugend danach verlangt, dem Land und der Gesellschaft in der sie leben, etwas zurückzugeben, auch ganz bewusst als Grundwehrdienstleistender. ${ }^{15}$

Die Gewinnung qualifizierten Nachwuchses für die Streitkräfte ist eine strategische Aufgabe für die Bundeswehr und stellt vor dem Hintergrund der demografischen Entwicklung eine zentrale Herausforderung dar.

Die allgemeine Wehrpflicht gewährleistet, dass das verfügbare Potenzial für die Nachwuchsgewinnung einen ausreichenden Umfang aufweist, um qualifizierten Nachwuchs für die Streitkräfte zu rekrutieren. Der Bundeswehr gelingt es bis dato, bis zu 40 Prozent seines Nachwuchses aus den Wehrpflichtigen zu gewinnen. ${ }^{16}$ Dabei sind vor allem auch Bewerber, die über die allgemeine Wehrpflicht den Beruf „Soldat" erst kennen und idealerweise schätzen lernen, um als Zeit- oder Berufssoldat Dienst zu leisten. Die allgemeine Wehrpflicht stellt damit einen Mehrwert für die Bundeswehr, aber auch für die

12 Vgl. dazu Wittmann, K. (2010). aaO, S. 7.

13 Vgl. dazu Buch, D. (2010). Die Zukunft der deutschen Wehrpflicht. Die Rückschlüsse aus dem europäischen Vergleich sprechen für eine dauerhafte Beibehaltung. Berlin, S. 18

14 Vgl. dazu Bischof, G. (2003). Statement des Referatsleiters FüS I 4 zum Thema „Wehrpflicht“. In: Prüfert, A. (Hrsg.), Hat die allgemeine Wehrpflicht in Deutschland eine Zukunft? Baden-Baden, S. 84/85.

15 Vgl. dazu Aussagen von Schülern im Rahmen des am 23.09.2008 durch den DBwV intiierten und durchgeführten Forums Wehrpflicht im Umweltforum Berlin. In Auszügen nachzulesen unter: http://www.bundeswehr.de/portal/ a/bwde/streitkraefte/wehrpflicht?yw_contentURL=/C1256EF4002AED30/ W27JRJRC933INFODE/content.jsp\#par5 (07.02.2010).

16 Vgl. dazu Jung, F.-J. (2008). Rede von Minister Jung anlässlich des Forums Wehrpflicht. http://www.bmvg.de/portal/a/bmvg/de/ministerium/geschichte_bmvg/verteidigungsminister_seit_1955/drfranzjosefjung?yw_contentURL=/C1256F1200608B1B/W27JREKY782INFODE/content.jsp, S. 3 (30.01.2010)
Grundwehrdienstleistenden dar, die die Chance erhalten, in der Bundeswehr ihre „Berufung“ zu finden. Zumal die Gewinnung von qualifiziertem Nachwuchs für die Nationen, welche die allgemeine Wehrpflicht ausgesetzt oder abgeschafft haben, ein Kernproblem im Bereich darstellt. Weiterhin sind in diesen Ländern deutlich höhere Aufwendungen im Bereich des Personals zu verzeichnen. ${ }^{17}$ Aber auch monetäre Anreize reichten beispielsweise in den USA und Großbritannien oftmals nicht aus, dringend benötigten Nachwuchs für ihre Streitkräfte zu rekrutieren. ${ }^{18}$

\section{c. Die allgemeine Wehrpflicht ist gesellschaft- lich wünschenswert}

Durch die allgemeine Wehrpflicht wird das wechselseitige Verhältnis von Gesellschaft und Streitkräften aufrechterhalten. Die Gesellschaft nimmt größeren Anteil an ihren Soldaten und deren Schicksal. Es wird einer Versicherungsmentalität in der Bevölkerung vorgebeugt, die Streitkräfte rein als bezahltes Instrument deutscher Interessenwahrung zu sehen. Die allgemeine Wehrpflicht fördert zudem die gewollte Integration der Soldaten in die Gesellschaft. Das Leitbild des „Staatsbürgers in Uniform“, das die Zielvorstellung der Konzeption der Inneren Führung versinnbildlicht, basiert dabei ganz wesentlich auf dieser Integration des Soldaten in die Gesellschaft und das „Getragen sein“ des Soldaten durch die Gesellschaft. ${ }^{19}$ Es ist die Frage zu stellen, ob nicht die viel zitierte und hoch geachtete Führungsphilosophie der Bundeswehr, die Innere Führung, ganz wesentlich auf die allgemeine Wehrpflicht abzielt? Einige Experten sehen diesen direkten Zusammenhang durchaus gegeben. ${ }^{20}$

Welche Art von Soldaten wollen wir in unseren Streitkräften haben und welchen Identitätsmustern sollen sie entsprechen? Diese Frage muss beantwortet werden, wenn man über die Wehrform diskutiert. Das Bild des archaischen Kämpfers steht hier dem sozial kompetenten, interkulturell gebildeten, intelligenten und leistungsbereiten Soldaten gegenüber. Unser Leitbild vom Staatsbürger in Uniform und die Grundsätze der Inneren Führung stellen hohe Anforderungen an die Soldatinnen und Soldaten der Bundeswehr. Die allgemeine Wehrpflicht hilft den Vorgesetzten dabei, diesen Anforderungen, auch im Friedensbetrieb, gerecht zu werden.

Die Grundwehrdienstleistenden mit ihren familiären Bindungen gewähren zudem weiten Teilen der Bevölkerung Einblick in ihre Streitkräfte. Aber auch die Bundeswehr wird durch die Wehrpflichtigen stets gefordert, sich aktuellen gesellschaftlichen Entwicklungen nicht zu verschließen und ihr eigenes Handeln zu überprüfen.

17 Vgl. dazu Buch, D. (2010), aaO., S. 48/51.

18 Vgl. dazu Sommer, T. (2003). Auswahlwehrpflicht und Wehrgerechtigkeit. In: Prüfert, A. (Hrsg.), Hat die allgemeine Wehrpflicht in Deutschland eine Zukunft? Baden-Baden, S. 62.

19 Vgl. dazu Bundesministerium der Verteidigung (2008). ZDv 10/1 Innere Führung. Selbstverständnis und Führungskultur der Bundeswehr. Bonn, S. $13 / 14$

20 Vgl. dazu Hoppe, T. (2001). Die Allgemeine Wehrpflicht - ein Auslaufmodell?. Sechs Thesen. http://www2.hsu-hh.de/thekat/hoppe-4.pdf, S. 3. 
Als verantwortungsbewusster Staatsbürger zu handeln, die Obligation des Bürgers für seinen Staat zu erfüllen, hieran hat die allgemeine Wehrpflicht wesentlichen Anteil. Die jungen Staatsbürger wollen diese Pflicht auch erfüllen, sie legen allerdings auch berechtigten Wert darauf, dass ihr Dienst sinnstiftend, weiterführend und gesellschaftlich anerkannt ist. ${ }^{21}$ Die allgemeine Wehrpflicht kann zudem wesentlich zur Persönlichkeitsbildung junger Staatsbürger beitragen. Zivilcourage, Verantwortungsbewusstsein, Toleranz und Kameradschaft sind bleibende Werte, die an die Grundwehrdienstleistenden vermittelt werden können und gesellschaftlich nachwirken.

Die Wahrnehmung sicherheitspolitischer Zusammenhänge erfolgt in der Gesellschaft in der Regel über die Medien. Dabei wird die Bundeswehr als Parlamentsarmee in der öffentlichen Debatte, aber auch in der medialen Wahrnehmung durch die allgemeine Wehrpflicht in besonderer Weise wahrgenommen. Die Grundwehrdienstleistenden tragen ganz wesentlich mit dazu bei, dass der verantwortungsbewusste Umgang der Politik mit dem Einsatz der Bundeswehr als international eingesetztes Mittel deutscher Außen- und Sicherheitspolitik gewahrt bleibt.

Aus den genannten Gründen hält der DBwV auch einen auf sechs Monate verkürzten Wehrdienst, wenn dieser attraktiv, sinnvoll und gerecht ausgestaltet ist, für unbedingt erstrebenswert. Allerdings muss diese Wehrpflicht dann angepasst werden. Zum einen muss sie den zu erwartenden strukturellen Veränderungen folgen und zum anderen muss sie inhaltlich den sicherheitspolitischen Erfordernissen angepasst werden. Diese Maßnahmen werden Zeit und Mühe, aber letztendlich auch Geld kosten.

Zusammenfassend gilt auch aus Sicht des DBwV die Prämisse, die jüngst der ehemalige Bundesaußenminister Hans-Dietrich Genscher, in einem Beitrag herausgestellt hat: „Auch als überzeugter Vertreter der Wehrpflichtarmee-Bundeswehr verkenne ich nicht, dass es durchaus Argumente für eine Abschaffung der Wehrpflicht gibt. Diese Argumente müssen sich mit den Fragen von grundsätzlicher Bedeutung auseinandersetzen. Die Haushaltszwänge für das Jahr 2011 und die folgenden Jahre geben die Antworten auf solche grundsätzlichen Fragen nicht. ... Bundesregierung und Deutscher Bundestag müssen sich auch in der gegenwärtigen Haushaltslage ihrer Verantwortung gegenüber den Soldaten bewusst sein.“22

21 Vgl. dazu Aussagen von Schülern im Rahmen des am 23.09.2008 durch den DBwV initiierten und durchgeführten Forums Wehrpflicht im Umweltforum Berlin. In Auszügen nachzulesen unter: http://www.bundeswehr.de/portal/ a/bwde/streitkraefte/wehrpflicht?yw_contentURL=/C1256EF4002AED30/ W27JRJRC933INFODE/content.jsp\#par5 (07.02.2010).

22 Der Tagesspiegel (2010). Wehrpflicht trotz Geldnot. http://www.tagesspiegel.de/meinung/wehrpflicht-trotz-geldnot/1853658.html?view=print (08.06.2010).

\section{Auswahlwehrpflicht als Alternative?}

Trotz der jüngsten Rechtsprechung höchster deutscher Gerichte oder aber der Nichtzulassung von Verfassungsbeschwerden zum Thema Wehrgerechtigkeit durch das Bundesverfassungsgericht bleibt das „Bauchgefühl“ beim Thema Gerechtigkeit in Fragen der allgemeinen Wehrpflicht bestehen. ${ }^{23}$ Dieses Unbehagen ist durchaus nachvollziehbar, wenn aktuell nur etwa 20 Prozent der jungen Männer eines Geburtsjahrgangs in die Streitkräfte einrücken. ${ }^{24}$

Einen Vorschlag, dieser aktuellen Situation gerecht zu werden, stellt die sogenannte Auswahlwehrpflicht dar. Diese Wehrform wurde bereits im Jahr 2000 von der „Weizsäcker-Kommission“ vorgeschlagen mit dem Ziel, die allgemeine Wehrpflicht, die man bereits damals als die beste Wehrform befand, beizubehalten. Die Kommission kam damals zu dem Schluss, dass die Auswahlwehrpflicht die Vorteile der Wehrpflicht für die Bundeswehr garantieren würde und gleichzeitig die notwendige Flexibilität bei einer am militärischen Bedarf orientierten Einberufung gewährleisten könnte. ${ }^{25}$

Gegen diese Art der Wehrform wurden und werden vielfach verfassungsrechtliche Bedenken angemeldet, da einige Kommentatoren das Gleichheitsgebot bei Anwendung einer rein am militärischen Bedarf orientierten Auswahlwehrpflicht verletzt sehen. Dieser Meinung hat sich der DBwV in einer ersten Reaktion nach Bekanntwerden des Vorschlags im Jahr 2000 weitgehend angeschlossen.

Doch muss in der aktuellen Debatte sicher konstatiert werden, dass die heute gültige Praxis der allgemeinen Wehrpflicht, mit einer Einberufungsquote der Geburtsjahrgänge 1975 bis 1986 von durchschnittlich etwa 29 Prozent der Männer, sicherlich ebenfalls eine gewisse Auswahl vornimmt. Auch wenn nach wie vor bei den Geburtsjährgängen 1975 bis 1986 durchschnittlich etwa 91,5 Prozent der tauglich gemusterten einen Pflichtdienst ableisten. $^{26}$

Sollten die verfassungsrechtlichen Bedenken gegenüber einer Auswahlwehrpflicht ausgeräumt werden können, müsste sicherlich das „Sonderopfer“, das die zum Wehrdienst Einberufenen erbringen, entsprechend honoriert werden. Darin wäre sicherlich auch eine Chance zu sehen, zum einen junge Männer für den Dienst am Staat zu motivieren und zum anderen den Beweis anzutreten, dass der Staat diesen Dienst auch entsprechend würdigt. In welcher Form diese Honorierung erfolgen würde, darüber müsste man sich verständigen. Der DBwV würde sich dieser Debatte allerdings sicher nicht verschließen.

23 Vgl. dazu Bundesverwaltungsgericht, VG 8 K 154/04 vom 19.01.2005 und Bundesverfassungsgericht, 2 BvL 3/09 vom 22.07.2009.

$24 \mathrm{Vgl}$. dazu Antwort der Bundesregierung auf eine Kleine Anfrage der Fraktion „Die Linke“. In: Bundestagsdrucksache 16/12522 vom 26.03.2009.

25 Vgl. dazu Kommission „Gemeinsame Sicherheit und Zukunft der Bundeswehr" (2000). Bericht der Kommission an die Bundesregierung. Bundesminsterium der Verteidigung. Berlin / Bonn, S. 68 f.

26 Vgl. dazu Buch, D. (2010). Die Wehrpflicht - gestern wie heute notwendig. In: Kirsch, U. (Hrsg.), Darum Wehrpflicht! Zur aktuellen Debatte um die Zukunft der deutschen Wehrpflicht. Baden-Baden, S. $47 \mathrm{ff}$. 